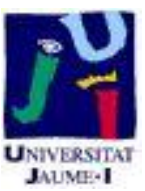

Título artículo / Títol article: Germanium coating boosts lithium uptake in Si nanotube battery anodes

Marta Haro, Taeseup Song, Antonio Guerrero

Autores / Autors

Castillejo, Luca Bertoluzzi, Juan Bisquert, Ungyu

Paik, Germà García Belmonte

Revista:

Physical Chemistry Chemical Physics, 2014, 16

Versión / Versió:

Post-print de l'autor

Cita bibliográfica / Cita bibliogràfica (ISO 690):
HARO, Marta, et al. Germanium coating boosts lithium uptake in Si nanotube battery anodes. Physical Chemistry Chemical Physics, 2014, vol. 16, no 33, p. 17930-17935.

http://hdl.handle.net/10234/122383 


\title{
Germanium coating boosts lithium uptake in Si nanotube battery anodes
}

\author{
Marta Haro ${ }^{1, \ddagger}$, Taeseup Song ${ }^{2, \ddagger}$, Antonio Guerrero ${ }^{1}$, Luca Bertoluzzi ${ }^{1}$, Juan Bisquert ${ }^{1}$, \\ Ungyu Paik $^{2}$, and Germà Garcia-Belmonte ${ }^{1 *}$
}

1. Photovoltaics and Optoelectronic Devices Group, Departament de Física, UniversitatJaume I, 12071 Castelló, Spain

2. Department of Energy Engineering, Hanyang University, Seoul 133-791, Korea

\begin{abstract}
Si nanotubes for reversible alloying reaction with lithium are able to accommodate large volume changes and offer improved cycle retention and reliable response when incorporated into battery anodes. However, $\mathrm{Si}$ nanotubes electrode exhibits poor rate capability because of its inherently low electron conductivity and Li ion diffusivity. $\mathrm{Si} / \mathrm{Ge}$ double-layered nanotubes electrode show promise to improve structural stability and electrochemical kinetics, as compared to homogeneous Si nanotube arrays. The mechanism explaining the enhancement in the rate capabilities is here revealed by means of electrochemical impedance methods. Ge shell efficiently provides electrons to the active materials which increase the semiconductor conductivity thereby assisting $\mathrm{Li}^{+}$ ion incorporation. The charge transfer resistance which accounts for the interfacial $\mathrm{Li}^{+}$ ion intake from the electrolyte is reduced by two orders of magnitude, implying the key role of Ge layer as electron supplier. Other resistive processes hindering the electrode charge/discharge process are observed to show comparable values for $\mathrm{Si}$ and $\mathrm{Si} / \mathrm{Ge}$ array electrodes.
\end{abstract}

Keywords: Semiconductor nanostructures, Li-ion batteries, Electrochemical kinetics, Rate capability, Electrochemical impedance spectroscopy

published in Phys. Chem. Chem. Phys. 2014, 16, 17930 


\section{Introduction}

Among various alloying-type anode materials for lithium ion batteries (LIB), Si has received considerable attention due to its highest theoretical capacity, $4200 \mathrm{mAh} / \mathrm{g}$ at the fully lithiated state $\mathrm{Li}_{22} \mathrm{Si}_{5}$, being the most promising alternative for carbon anodes $[1,2]$. Although the fast capacity fading of $\mathrm{Si}$ electrode (resulting from large volume change associated with lithium ion) has been considered as a main obstacle for its practical use, significant improvement in the cycle performance has been achieved by engineering the geometry and dimension of Si anode materials [3, 4]. Especially, Si nanotubes (Si NT) array exhibited the robust cyclability due to the reversible morphological change [5-7]. Electrode materials for LIB should be designed to fulfill both energy density and power density requirements of critical applications such as large-scale storage for renewable power sources, electric vehicles, and plug-in hybrid electric vehicles. However, Si NT electrode could not meet the demand on the high power density due to its poor rate capability attributed to inherently low electron conductivity and ion diffusivity. To improve these two parameters researchers have investigated in the last years different strategies such as the growth of $\mathrm{Si}$ on nanopillar metallic structrures [8], fabrication of core-shell composites [9], or coating of Si electrode with a good electron and/or ion conductor (polymer, graphene, etc) [10-14]. Very recently, Si/Ge double-layered nanotubes ( $\mathrm{Si} / \mathrm{Ge} \mathrm{DLNT}$ ) array prepared by employing a template-assisted synthesis method based on chemical vapor deposition process has been reported [15]. With optimal designs, Si/Ge DLNT exhibited significant improvements in structural stability and electrochemical kinetics in comparison to homogeneous Si NT. Although it is evident that high electronic conductivity and ionic diffusivity in $\mathrm{Ge}$ in comparison to $\mathrm{Si}$ aid to explain the kinetic improvements, the exact mechanism (either electronic conduction, ionic migration, charge transfer, or alloying reaction) that lies behind such an enhancement is still unclear [15-17].

We conclude by means of impedance spectroscopy measurements that the incorporation of Li-ions from the electrolyte into the semiconductor structures is highly favored by the Ge shell covering inner Si nanotubes. This is principally related to the higher electronic conductivity that Ge possesses in comparison to $\mathrm{Si}$. The analysis of the resistive processes hindering the electrode charging points to a kinetic limitation in the case of Si NT related to interfacial mechanisms. The total resistance for Si NT electrode doubles that encountered for $\mathrm{Si} / \mathrm{Ge}$ DLNT structure in the range of the alloying reaction potentials. The rate capability is then enhanced by incorporating the Ge shell that reduces as a consequence of the charge transfer resistance associated to the $\mathrm{Li}$-ion intake.

\section{Results and discussion}

\subsection{Electrochemical properties}

Experimental details for the synthesis and evaluation of electrochemical properties of both the Si NT and the Si/Ge DLNT anodes has been fully addressed in previous reports 
[7, 15]. Figure 1 (a) and (b) display low magnification transmission electron microscopy (TEM) images of Si NT and Si/Ge DLNT. Both Si NT and Si/Ge DLNT show tubular morphology and identical dimensions. The Si NT has the outer diameter of $\sim 120 \mathrm{~nm}$ and shell thicknesses of $\sim 30 \mathrm{~nm}$. In Si/Ge DLNT, a Ge shell layer with a thickness ranging from 10 to $15 \mathrm{~nm}$ is uniformly coated on the surface of a SiNT with a thickness ranging from 15 to $20 \mathrm{~nm}$ and the inner diameter of $\sim 600 \mathrm{~nm}$.
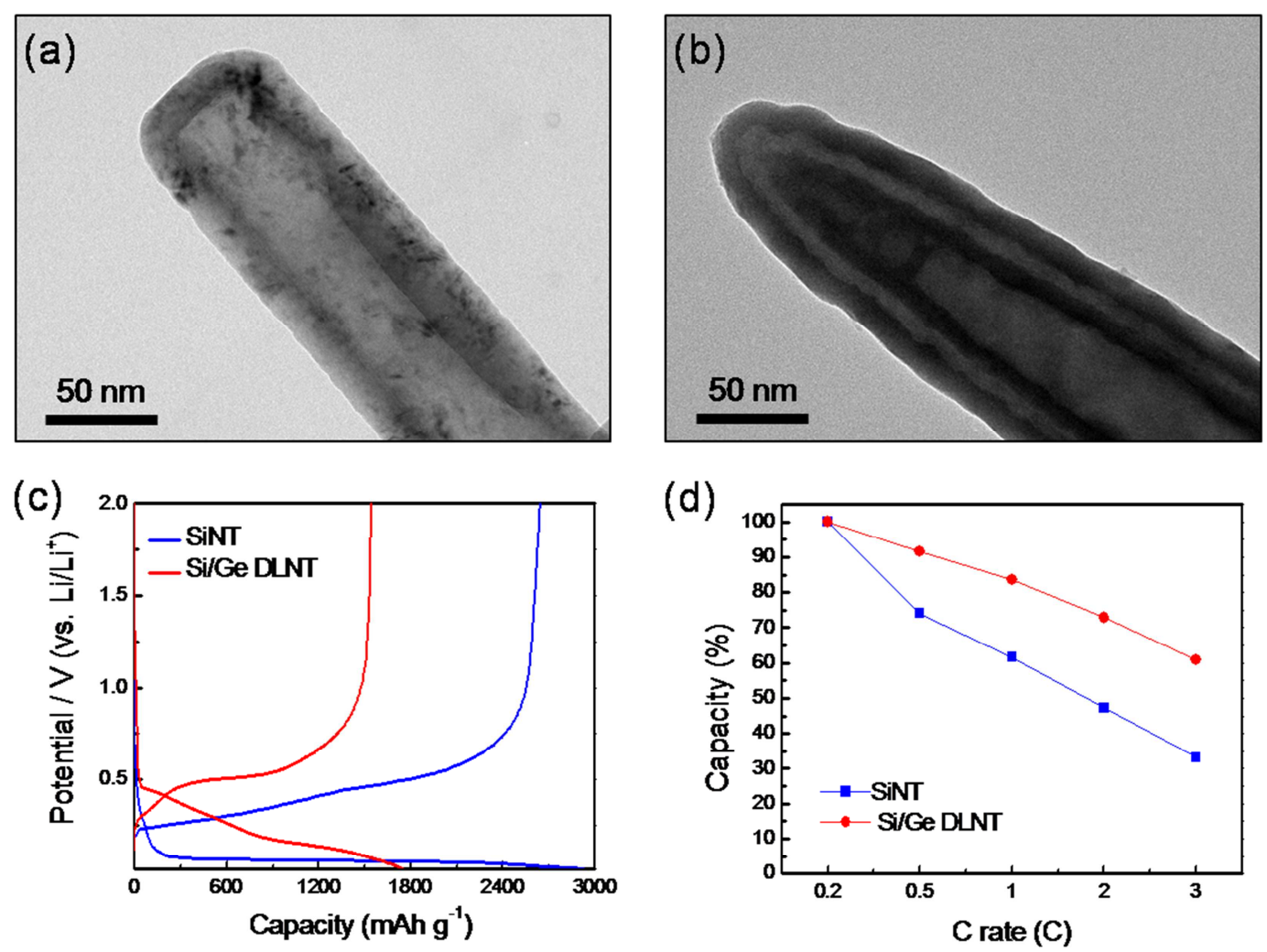

Figure 1. TEM images of SiNT (a) and Si/Ge DLNT (b). The first voltage profiles at a rate of $0.2 \mathrm{C}$ (c) and rate capabilities (d) for SiNT and Si/Ge DLNT electrodes.

The electrochemical performances of both Si NT and Si/Ge DLNT electrodes were evaluated over the potential window of $0 \sim 2.0 \mathrm{~V} \mathrm{vs.} \mathrm{Li} / \mathrm{Li}^{+}$at room temperature $\left(25^{\circ} \mathrm{C}\right)$. Figure 1 (c) shows the voltage profiles at the first cycle for Si NT and Si/Ge DLNT electrodes. Although Si NT electrode $(\sim 2650 \mathrm{mAh} / \mathrm{g})$ delivered $\sim 2$-fold higher gravimetric capacity than that of the $\mathrm{Si} / \mathrm{Ge}$ DLNT electrode $(\sim 1550 \mathrm{mAh} / \mathrm{g}), \mathrm{Si} / \mathrm{Ge}$ DLNT electrode $\left(\sim 1.2 \mathrm{mAh} / \mathrm{cm}^{2}\right)$ exhibited 2-fold higher areal capacity compared to that of the Si NT electrode $\left(\sim 0.6 \mathrm{mAh} / \mathrm{cm}^{2}\right)$ along with improved cycle performance. The Si NT and Si/Ge DLNT electrodes showed totally different voltage profiles during charging (lithium insertion). Si NT electrode shows a long plateau near $0.1 \mathrm{~V}$, which corresponds to the alloying reaction of the crystalline $\mathrm{Si}$ with lithium. The Si/Ge DLNT array showed two distinct voltage plateaus in the discharge voltage profile. The first 
voltage plateau, located between 0.2 and $0.4 \mathrm{~V}$, and second downward slope voltage plateau, located under $0.2 \mathrm{~V}$, are attributed to the alloying reactions of Ge shell layer and $\mathrm{Ge} / \mathrm{Si}$ with lithium, respectively. The rate capability of $\mathrm{Si}$ NT and $\mathrm{Si} / \mathrm{Ge}$ DLNT electrodes were carefully evaluated at various C-rates (Figure 1 (d). Si/Ge DLNT electrode exhibits significantly improved rate capability compared to that of Si NT electrode. While Si/Ge DLNT electrode retains the capacity over $60 \%$ at $3 \mathrm{C}$ rate, $\mathrm{Si}$ NT electrode delivers very low capacity $(35 \%)$. This significant improvement in rate capability observed for Si/Ge DLNT might be related to several steps involved in the lithiation/delithitiation process. To further understand the kinetics of Li-ion intake and subsequent alloying reaction anodes are investigated by using electrochemical impedance spectroscopy (EIS) methods.

\subsection{Impedance responses}

Kinetic limitations to the charging/discharging process have been addressed by analyzing EIS response performed at open-circuit voltage (no direct current flowing) at different charge states between $0 \mathrm{~V}$ and $1.2 \mathrm{~V}$, with amplitude of $20 \mathrm{mV}$ in the frequency range from $10^{6} \mathrm{~Hz}$ down to $0.001 \mathrm{~Hz}$. Kinetic limitations can be easily inferred from resistive processes occurring during the electrode operation in steady-state conditions. All the results are normalized by the weight for a clearer comparison. Representative Nyquist plots are represented in Fig. 2, which consist of two welldefined parts: the high-frequency semicircles, and an inclined low-frequency capacitivelike line. At high frequencies, a rather constant, minor arc corresponds to the parallel connection between the interlayer capacitance $C_{\mathrm{il}} \approx 2-70 \mu \mathrm{F} \mathrm{mg}^{-1}$ and migration resistance $R_{\mathrm{il}} \approx 2-10 \Omega \mathrm{mg}$ (Fig. $2 \mathrm{~b}$ ). This resistive contribution is usually caused by the Li migration through external layers as those formed by the solid electrolyte interface (SEI) associated with the electrolyte decomposition (see Fig. 3a). This impedance contribution is significantly smaller than the additional arc observed at intermediate-frequencies, clearly visible in the case of Si NT electrodes, which is interpreted in terms of the mechanisms occurring at the electrical double-layer formed at the nanotubes (Fig. 3a). The intermediate process is modeled by means of the doublelayer capacitance $C_{\mathrm{dl}}$ and charge transfer resistance $R_{\mathrm{ct}}$. This last resistance is related to the Li-ion intake step to the nanotubes. Huge difference is observed in the charge transfer resistance $R_{\mathrm{ct}}$ corresponding to the intermediate arc when $\mathrm{Si}$ and $\mathrm{Si} / \mathrm{Ge}$-based electrodes are compared. While Si NT electrodes exhibit an increase in $R_{\mathrm{ct}}$ when potential is reduced below $0.5 \mathrm{~V}\left(R_{\mathrm{ct}} \approx 200 \Omega \mathrm{mg}\right)$, the incorporation of Ge layer reduces the charge transfer resistance to values as low as $R_{\mathrm{ct}} \approx 3 \Omega \mathrm{mg}$ as observed in Fig. 2b. The porosity of the electrodes yields double-layer capacitance values in the order of $C_{\mathrm{dl}} \approx 10 \mu \mathrm{F} \mathrm{mg}^{-1}$. A connection between the impedance patterns and the corresponding circuit elements (Fig. 3a) can be viewed in Fig. 2c. This equivalent circuit accounts for the high-frequency impedance response with the parameters $R_{\mathrm{il}}, R_{\mathrm{ct}}, C_{\mathrm{il}}$, 
and $C_{\mathrm{dl}}$. A small series resistance accounts for the solution contribution (not shown in Fig. 3a).
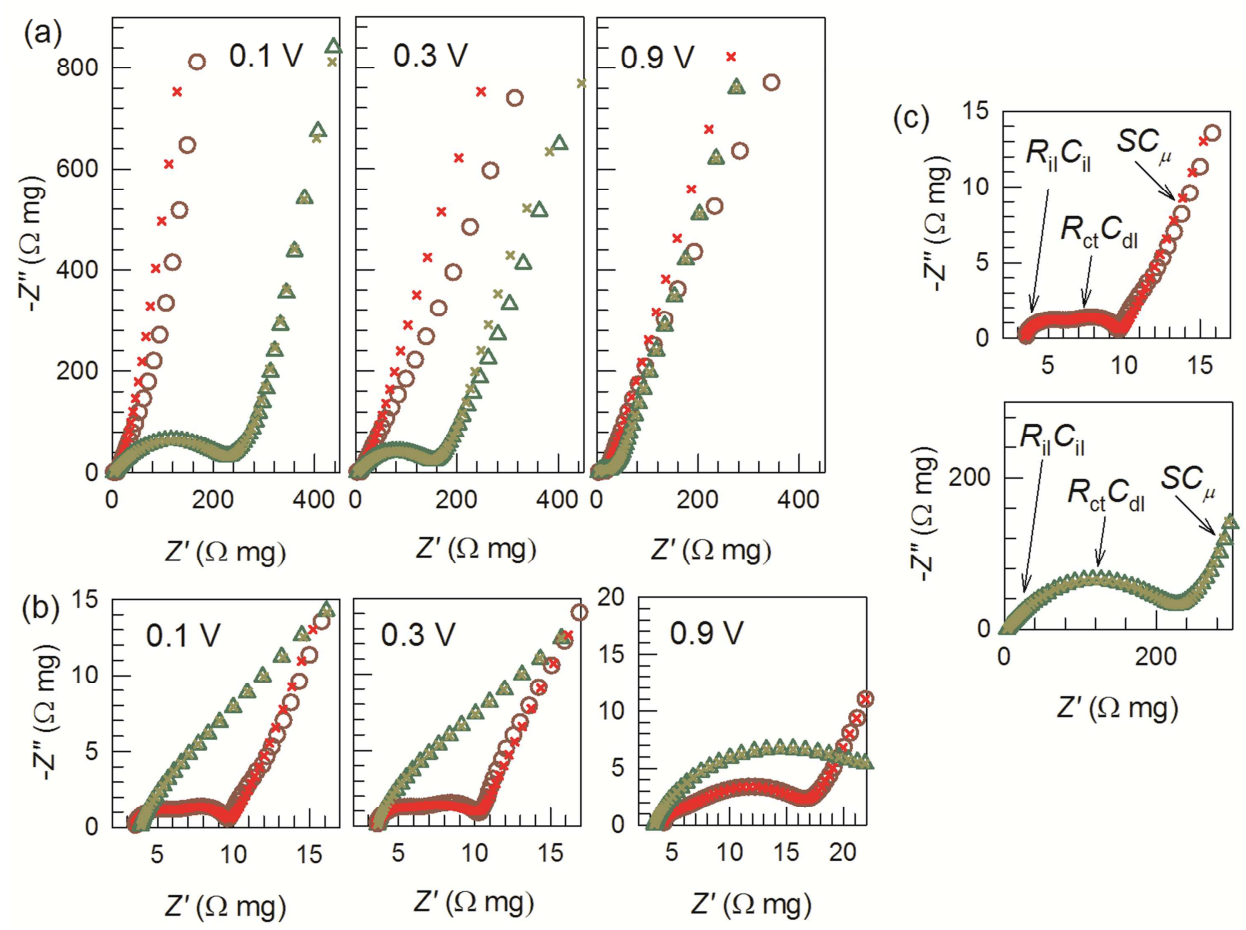

Figure 2. Impedance spectroscopy response of $\mathrm{Si} \mathrm{NT}$ (circle) and $\mathrm{Si} / \mathrm{Ge} \mathrm{DLNT}$ (triangle) NT electrodes at different steady-state voltages as indicated. (a) Complete view showing low-frequency capacitive-like response. (b) High-frequency detail with the interfacial behavior. Experimental data and fits (cross) using the equivalent circuit Figure 3a. (c) Impedance patterns at $0.1 \mathrm{~V}$ in relation with the equivalent circuit elements of Figure 3a. 

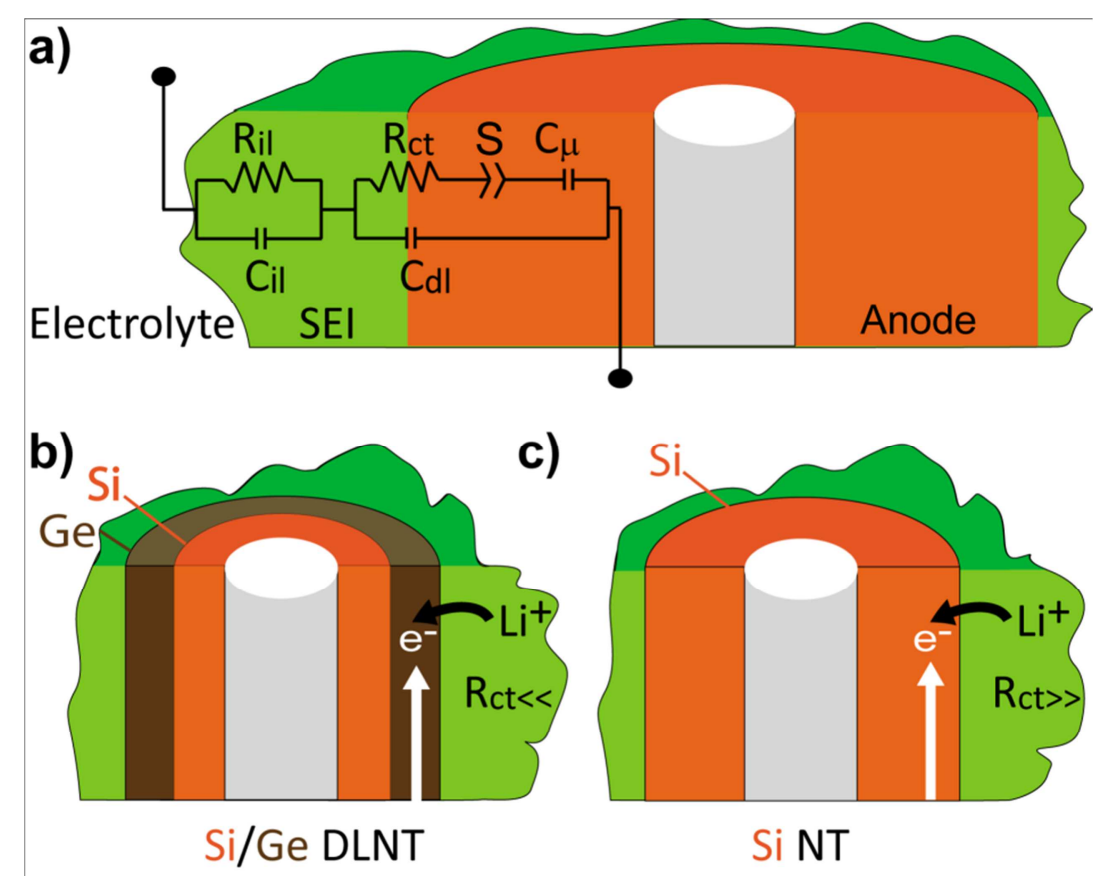

Figure 3.(a) Schematic view of the relation between equivalent circuit elements and electrode layers. $R_{\mathrm{il}}$ and $C_{\mathrm{il}}$ accounts for high-frequency processes occurring at the outer SEI layer. Interfacial mechanisms are modeled with $R_{\mathrm{ct}}$ and $C_{\mathrm{dl}}$ circuit elements. In series with $R_{\mathrm{ct}}$ the alloying reaction subcircuit includes the elements $S$ and $C_{\mu}$. (b) Favored electron conduction through Ge shell assists the Li-ion intake reducing $R_{\mathrm{ct}}$. (c) Difficult conduction in Si layeryields large values for $R_{\mathrm{ct}}$.

The high-frequency circuit elements are observed to change at potentials approaching alloying reactions, then signaling that mechanisms occurring at the interface are influenced by the electrode state of charge. It is worth noting here that the resistive parameter that exhibits the main difference between Si NT and Si/Ge DLNT electrodes is the charge transfer resistance $R_{\mathrm{ct}}$, which is reduced in more than two orders of magnitude. This resistance is connected to the $\mathrm{Li}$ intake from the solution to the semiconductor layer as schematically displayed in Fig. 3b-c. Ge shell allows for an enhanced electron path then facilitating the Li-ion intake at the semiconductor interface. It has been observed for other insertion compounds that $R_{\mathrm{ct}}$ is directly related to the electronic conductivity of the host material [18]. High conductive hosts permit the Li ion to overcome the potential barrier appearing at the solution/semiconductor interface. In the case under study it is known that Ge electronic conductivity exceeds in four orders of magnitude that encountered for $\mathrm{Si}$. This fact explains the observed reduction in $R_{\mathrm{ct}}$ for potentials in the alloying reaction range. Alternatively the intermediate arc resistance might be also directly related to the electronic conductivity of the semiconductor. But in this last case it is hard to understand how $R_{\mathrm{ct}}$ presents values in the range of 100-200 $\Omega$ $\mathrm{mg}$ for both electrodes at potentials in excess of $0.5 \mathrm{~V}$ (see Fig. 2b). This fact leads us to connect the limiting lithiation mechanism represented by $R_{\mathrm{ct}}$ to the process of Li-ion incorporation into the semiconductor matrix. 


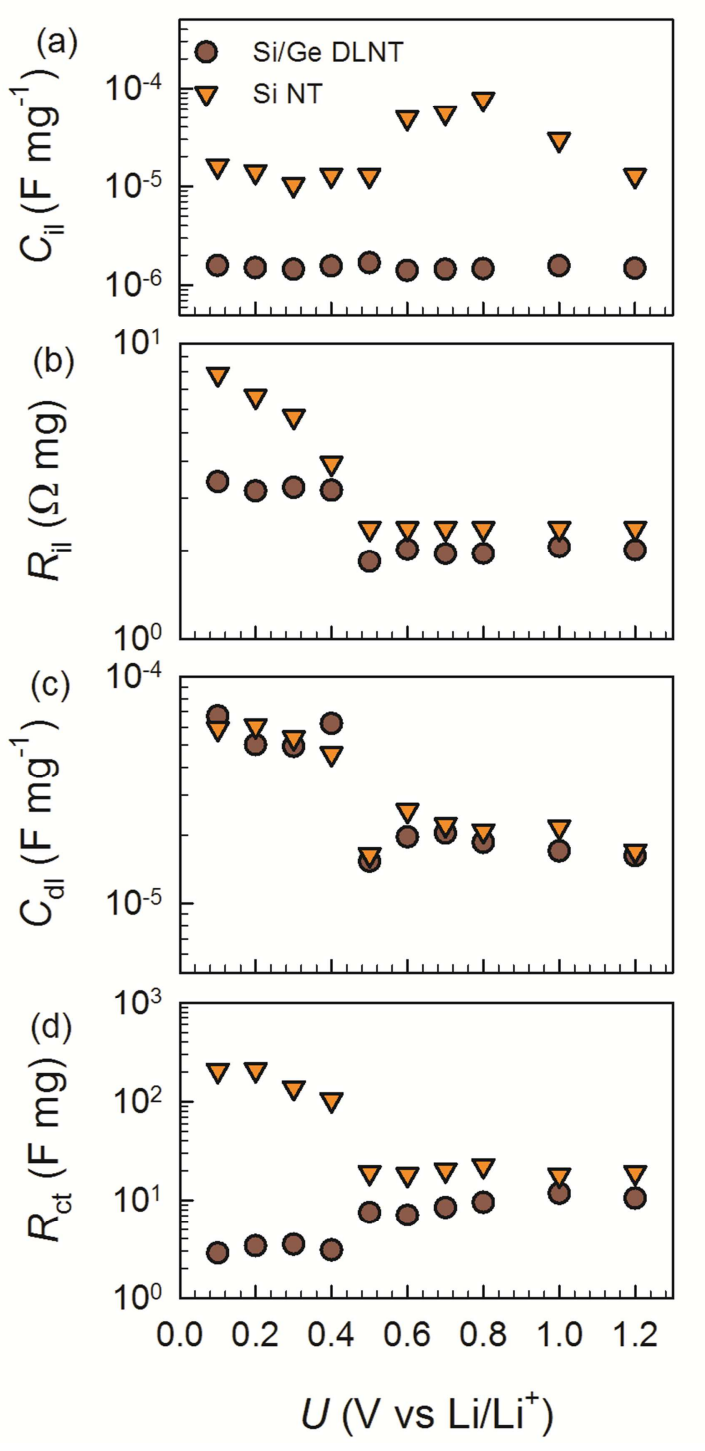

Figure 4. Fitting results using the equivalent circuit of Fig. 3(a) for the high-frequency circuit elements of $\mathrm{Si}$ and $\mathrm{Si} / \mathrm{Ge} \mathrm{NT}$ anodes showing (a) interlayer capacitance $C_{\mathrm{il}}$ and (b) migration resistance $R_{\mathrm{il}}$. (c)Double-layer capacitance $C_{\mathrm{dl}}$, and (d) charge transfer resistance $R_{\mathrm{ct}}$.

Parameter values drawn in Fig. 4a-d result from fitting the equivalent circuit of Fig. $3 a$ to the experimental impedance data. It is especially interesting to note the behavior exhibited by the charge transfer resistance (Fig. 4d) as it corresponds to the main hindrance mechanism for Li-ion supply to the alloying compound. For potentials below $0.5 \mathrm{~V} \mathrm{Si} \mathrm{NT}$ arrays undergo an increase in $R_{\mathrm{ct}}$ of one order of magnitude (from $\sim 20 \Omega$ $\mathrm{mg}$ up to $\sim 200 \Omega \mathrm{mg}$ ). On the contrary $\mathrm{Si} / \mathrm{Ge}$ DLNT exhibits the expected variation: as the system reaches the alloying potentials Ge conductivity is enhanced then reducing $R_{\mathrm{ct}}$. We can conjecture that this dissimilar behavior might be related to the different doping character of Si and Ge layers, although more investigations are needed on this 
concern that lies outside the scope of this work.

The low-frequency EIS response points to the occurrence of a voltage-modulated process which gives rise to a capacitive-like behavior. This behavior is connected to the so-called chemical capacitance that results from the electrode ability of varying the amount $c$ of reacted $\mathrm{Li}^{+}$upon application of a differential change in the chemical potential $\mu$ (directly assimilated to the electrode potential upon steady-state conditions as $\mu=q U$, where $q$ is the positive elementary charge) [19]. The chemical capacitance can be written as

$$
C_{\mu}=q \frac{d c}{d U}
$$

Accompanying the chemical capacitance at low-frequencies it is observed that a concomitant increase in the real part of the impedance appears added to the previous resistances acting at higher-frequencies (Fig. 2a). It is noticeable that this extra resistance at low-frequencies $R_{\mathrm{a}}$ exhibits similar values for $\mathrm{Si} \mathrm{NT}$ and $\mathrm{Si} / \mathrm{Ge}$ DLNT electrodes. As previously commented, resistances in the equivalent circuit signal the occurrence of different rate-limiting mechanisms. For intercalation compounds the diffusion of $\mathrm{Li}^{+}$inside the host material is one of the rate-limiting process [20]. Diffusion of ions gives rise to distinctive impedance patterns characterized by Warburglike responses as $Z \propto(i \omega)^{-1 / 2}$ (being $\omega$ the angular frequency, and $i=\sqrt{-1}$ ). Previous models based on spatially-restricted ion diffusion were proposed relying on a distribution of diffusion lengths ${ }^{\text {[21], }}$ or electronic transport limitations [22]. However, Si NT and Si/Ge DLNT electrodes function by alloying reactions yielding a complete chemical and structural electrode material rearrangement. The fact that no Warburg-like pattern is observed in the low-frequency response of Fig. 2a lead us to regard the alloying reaction itself as the origin for the additional resistance at low frequencies. The resistive process $R_{\mathrm{a}}$ accompanying the chemical capacitance is seen then as a contribution to the alloying-related current hindrance [23].

The coupling between chemical capacitance $C_{\mu}$ and reaction resistance $R_{\mathrm{a}}$ has been addressed in previous works [24]. In terms of equivalent circuits the alloying reaction can be modeled by means of a series connection of $C_{\mu}$ and a suitable generalization of $R_{\mathrm{a}}$ (a constant phase element CPE with impedance $Z_{\mathrm{CPE}}=1 / S(i \omega)^{\alpha}$ ) as drawn in the equivalent circuit of Fig. 3a. The effective alloying resistance can be calculated from fitting parameters by means of the next expression [24],

$$
R_{\mathrm{a}}=\left(C_{\mu}^{\alpha} / S\right)^{\frac{1}{1-\alpha}}
$$

Here $S$ accounts for the CPE parameter and $\alpha$ informs on the broadening in the reaction rate distribution. Figure 5 summarizes the fitting results. It is observed in Fig. 5a that the chemical capacitance $C_{\mu}$ steeply increases toward low potentials. $C_{\mu}$ values are obtained in the order of $0.1 \mathrm{~F} \mathrm{mg}^{-1}$ at low voltages for both electrodes informing about similar alloying ability of both $\mathrm{Si}$ and $\mathrm{Si} / \mathrm{Ge}$ semiconductors. It is interesting to 
point out that the chemical capacitance correlates with the discharge curve derivative $-d Q / d U$. Recalling now Fig. 1c, a long plateau is observed for Si NT electrodes at low voltages. This is in good agreement with the $C_{\mu}$ maximum at $0.1 \mathrm{~V}$. On the contrary Si/Ge DLNT discharge produces two distinctive plateaus at $\sim 0.5 \mathrm{~V}$ and $\sim 0.2 \mathrm{~V}$ that correspond well to the two $C_{\mu}$ maxima observed in Fig. $5 \mathrm{a}$ at $0.4 \mathrm{~V}$ and $0.1 \mathrm{~V}$. As inferred from TEM images and SAED patterns [15], the first maximum at $0.4 \mathrm{~V}$ is linked with the lithiation of the Ge shell, while the feature at $0.1 \mathrm{~V}$ arises from the alloying reaction of both $\mathrm{Ge}$ and $\mathrm{Si}$.

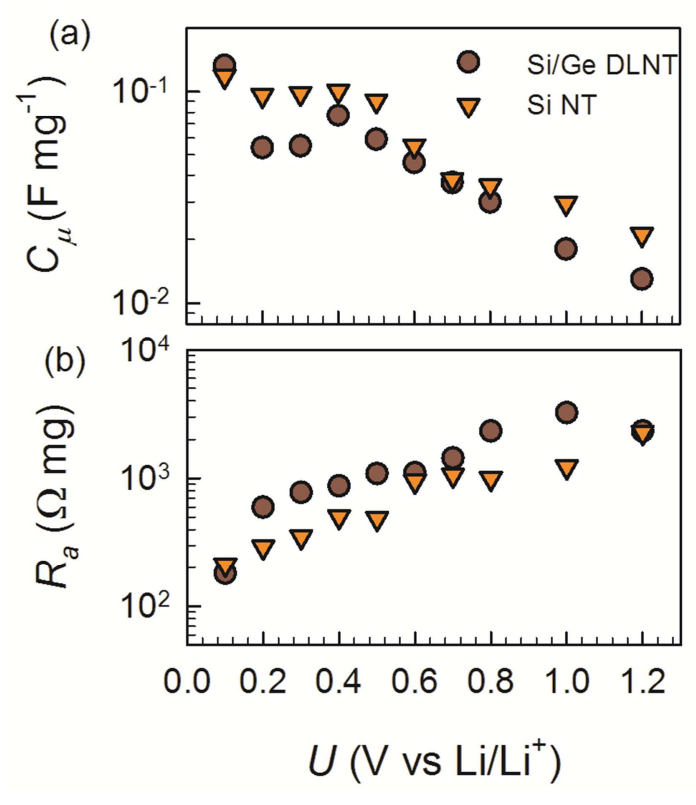

Figure 5.Fitting results using the equivalent circuit of Fig. 3(a) for the low-frequency circuit elements of $\mathrm{Si}$ and $\mathrm{Si} / \mathrm{Ge} \mathrm{NT}$ anodes showing (a) chemical capacitance $C_{\mu}$ and (b) alloying resistance $R_{\mathrm{a}}$.

The parameter accounting for the electrochemical kinetics is the alloying resistance of Eq. (2). One can infer from Fig. $5 \mathrm{~b}$ that $R_{\mathrm{a}}$ exhibits similar values for both Si NT and $\mathrm{Si} / \mathrm{Ge}$ DLNT electrodes at low potentials $(0.1 \mathrm{~V})$. At higher potentials $(0.2-0.6 \mathrm{~V})$ a slightly larger resistance is extracted for $\mathrm{Si} / \mathrm{Ge}$ DLNT electrodes. It is known that specific capacity at different C-rates can be also analyzed in terms of the resistive mechanisms accompanying the charging/discharging process. As shown in Fig. 1d, noticeable capacity decrease for the $\mathrm{Si}$ NT electrode was observed at $0.5 \mathrm{C}$. This is caused by a significant increase in the total resistance that at $0.1 \mathrm{~V}$ approximately amounts $200 \Omega \mathrm{mg}$ for Si/Ge DLNT electrodes, while that value doubles (400 $\Omega \mathrm{mg}$ ) in the case of Si NT. For higher charging/discharging rates high-frequency resistances $R_{\mathrm{il}}+R_{\mathrm{ct}}$ take the control of the kinetic response. In this case the different between $\mathrm{Si}$ NT (270 $\Omega \mathrm{mg}$ ) and $\mathrm{Si} / \mathrm{Ge}$ DLNT (50 $\Omega \mathrm{mg}$ ) resistive response is even larger. We 
remark again that the main contribution for increasing the Li-ion intake hindrance is caused by the superior charge transfer resistance exhibited by Si NT electrodes. This last observation clarifies the origin in the difference of the rate capability.

With the aim of further illustrating the detrimental effect of resistive elements on the C-rate performance, we have reproduce a simplified version of the discharge curve based on the equivalent circuit in Figure 3(a). In order to assess the relative importance of the extraction current, $I_{0}$, and charge transfer resistance, $R_{\mathrm{ct}}$, on the voltage decay, the electrode has been modeled by using the electrical circuit of Fig. 6(a). This circuit only takes into account the largest capacitive contribution. For the sake of simplicity chemical capacitance has been selected as a constant, while $R_{\mathrm{a}}$ follows the decreasing behavior with voltage shown in Fig. 5(b). The total specific charge in each simulation is extracted for the time at which potential attains zero. The potential drop at the resistive elements increases with the discharging current so leaving non-extracted charge in the capacitor. The simulation roughly reproduces the voltage decay and capacity plots of Figs. 1(c) and (d), as depicted by Figs. 6(b) and (c). The detailed procedure of these simulations is given in SI. As expected, higher specific current produces faster voltage fall, while larger charge transfer resistances are responsible for higher decrease in the capacity of the system. Doubling the resistance approximately reproduces the reduction in capacity observed in Figure 1(d) at 3C rate evaluation. Although the simulation is certainly an oversimplification of a real battery electrode, it highlights the role of resistive elements on the overall rate capability performance. 
(a)

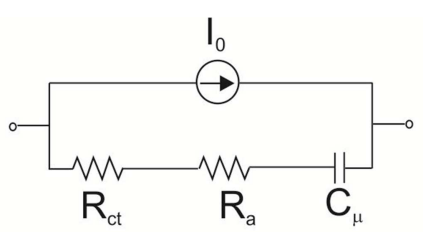

(b)
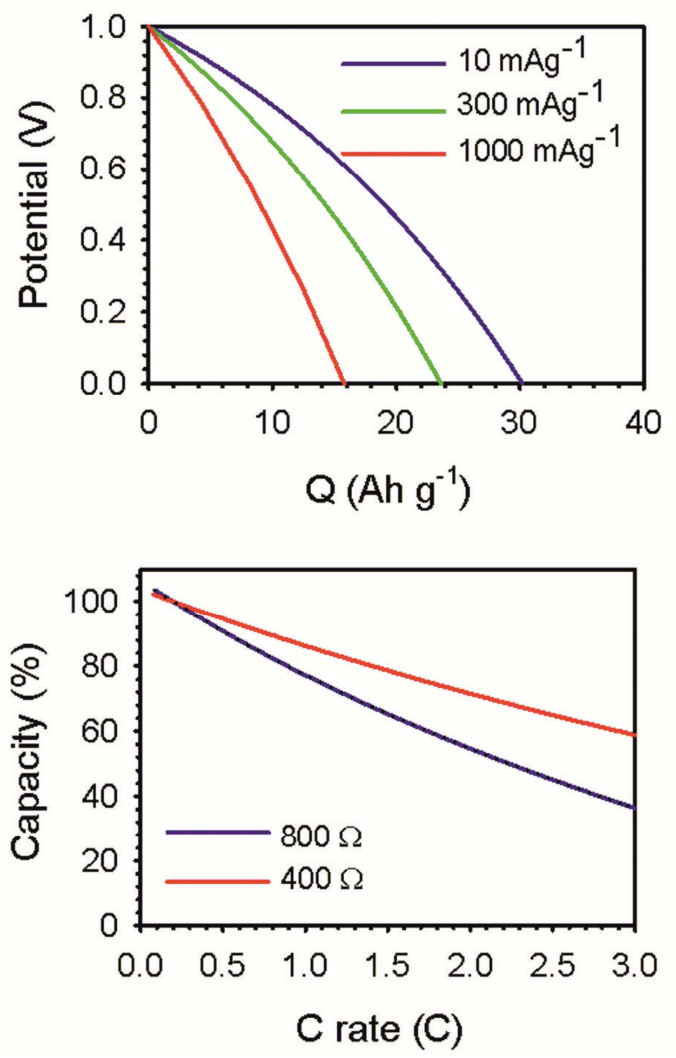

Figure 6.(a) Simplified equivalent circuit used to model the voltage decay in the discharge experiment. $I_{0}$ is the extraction current, $R_{\mathrm{ct}}$ is the charge transfer resistance, $R_{\mathrm{a}}$ is the alloying resistance and $C_{\mu}$ is the chemical capacitance. (b) Voltage decay for different values of the specific current, $I_{0}$, indicated in the inset panel. The charge transfer resistance was fixed at the value $R_{\mathrm{ct}}=800 \Omega$. (c) Evolution of the capacity as a function of the current rate for two values of the charge transfer resistance (indicated in the inset panel of this figure).

\section{Conclusions}

We have identified the mechanism that causes the difference in rate capability between Si NT and Si-Ge DLNT electrodes for Li-ion battery anodes. It is observed by impedance spectroscopy that the intake of Li-ions from the electrolyte into the active materials is highly favored by the Ge shell covering inner Si nanotubes. This is mainly related to the higher electronic conductivity that Ge possesses in comparison to Si. The total resistance for $\mathrm{Si}$ NT electrodes doubles that encountered for $\mathrm{Si} / \mathrm{Ge}$ DLNT structures. 


\section{Author information}

${ }^{\ddagger}$ These authors contributed equally to this work.

Corresponding Author

*E-mail: upaik@hanyang.ac.kr (U. Paik), garciag@fca.uji.es (G.G.-B.).

The authors declare no competing financial interest.

\section{Acknowledgments}

We thank financial support from Generalitat Valenciana (ISIC/ 2012/008 Institute of Nanotechnologies for Clean Energies) and from the European Union Seventh Framework Program (FP7/2007-2013) under project DESTINY (grant agreement 316494) Future Planning, an International Cooperation program of the Korea Institute of Energy Technology Evaluation and Planning (KETEP) grant funded by the Korean Ministry of Trade, Industry \& Energy (2011T100100369) is also acknowledged. 


\section{References}

[1] Song, T.;Hu, L. B.; Paik, U. One-Dimensional Silicon Nanostructures for Li Ion Batteries. J Phys Chem Lett 2014, 5, 720-731.

[2] Kasavajjula, U.;Wang, C. S.; Appleby, A. J. Nano- and bulk-silicon-based insertion anodes for lithium-ion secondary cells. J Power Sources 2007, 163, 10031039.

[3] Wu, H.; Cui, Y. Designing nanostructured Si anodes for high energy lithium ion batteries. Nano Today 2012, 7, 414-429.

[4] Szczech, J. R.; Jin, S. Nanostructured silicon for high capacity lithium battery anodes. Energ Environ Sci 2011, 4, 56-72.

[5] Chan, C. K.;Peng, H.;Liu, G.;McIlwrath, K.;Zhang, X. F.;Huggins, R. A.; Cui, Y. High-performance lithium battery anodes using silicon nanowires. Nature Nanotechnology 2008, 3, 31-35.

[6] Xiao, Y.;Hao, D.;Chen, H.;Gong, Z.; Yang, Y. Economical Synthesis and Promotion of the Electrochemical Performance of Silicon Nanowires as Anode Material in Li-Ion Batteries. ACS Applied Materials \& Interfaces 2013, 5, 1681-1687.

[7] Song, T.;Xia, J. L.;Lee, J. H.;Lee, D. H.;Kwon, M. S.;Choi, J. M.;Wu, J.;Doo, S. K.;Chang, H.;Il Park, W.;Zang, D. S.;Kim, H.;Huang, Y. G.;Hwang, K. C.;Rogers, J. A.; Paik, U. Arrays of Sealed Silicon Nanotubes As Anodes for Lithium Ion Batteries. Nano Lett 2010, 10, 1710-1716.

[8] Kim, G.;Jeong, S.;Shin, J.-H.;Cho, J.; Lee, H. 3D Amorphous Silicon on Nanopillar Copper Electrodes as Anodes for High-Rate Lithium-Ion Batteries. ACS Nano 2014.

[9] Jeong, G.;Kim, J.-G.;Park, M.-S.;Seo, M.;Hwang, S. M.;Kim, Y.-U.;Kim, Y.J.;Kim, J. H.; Dou, S. X. Core-Shell Structured Silicon Nanoparticles@ TiO2-x/Carbon Mesoporous Microfiber Composite as a Safe and High-Performance Lithium-Ion Battery Anode. ACS Nano 2014, 8, 2977-2985.

[10] Chen, Y.;Zeng, S.;Qian, J.;Wang, Y.;Cao, Y.;Yang, H.; Ai, X. Li+-Conductive Polymer-Embedded Nano-Si Particles as Anode Material for Advanced Li-ion Batteries. ACS Applied Materials \& Interfaces 2014, 6, 3508-3514.

[11]Jo, M. R.;Heo, Y.-U.;Lee, Y. C.; Kang, Y.-M. A nano-Si/FeSi 2 Ti heterostructure with structural stability for highly reversible lithium storage. Nanoscale 2014, 6, 1005-1010.

[12] Kim, W.-S.;Hwa, Y.;Shin, J.-H.;Yang, M.;Sohn, H.-J.; Hong, S.-H. Scalable synthesis of silicon nanosheets from sand as an anode for Li-ion batteries. Nanoscale 2014. 
[13] Wu, P.;Wang, H.;Tang, Y.;Zhou, Y.; Lu, T. Three-Dimensional Interconnected Network of Graphene-Wrapped Porous Silicon Spheres: In Situ Magnesiothermic Reduction Synthesis and Enhanced Lithium Storage Capabilities. ACS Applied Materials \& Interfaces 2014.

[14] Yue, C.;Yu, Y.;Wu, Z.;He, X.;Wang, J.;Li, J.;Li, C.;Wu, S.;Li, J.; Kang, J. Enhanced reversible lithium storage in germanium nano-island coated 3D hexagonal bottle-like Si nanorod arrays. Nanoscale 2014, 6, 1817-1822.

[15] Song, T.;Cheng, H.;Choi, H.;Lee, J.-H.;Han, H.;Lee, D. H.;Yoo, D. S.;Kwon, M.-S.;Choi, J.-M.;Doo, S. G.;Chang, H.;Xiao, J.;Huang, Y.;Park, W. I.;Chung, Y.C.;Kim, H.;Rogers, J. A.; Paik, U. Si/Ge Double-Layered Nanotube Array as a Lithium Ion Battery Anode. ACS Nano 2012, 6, 303-309.

[16] Song, T.;Cheng, H.;Town, K.;Park, H.;Black, R. W.;Lee, S.;Park, W. I.;Huang, Y.;Rogers, J. A.;Nazar, L. F.; Paik, U. Electrochemical Properties of Si-Ge Heterostructures as an Anode Material for Lithium Ion Batteries. Advanced Functional Materials 2014, 24, 1458-1464.

[17] Yu, Y.;Yue, C.;Sun, S.;Lin, W.;Su, H.;Xu, B.;Li, J.;Wu, S.;Li, J.; Kang, J. The Effects of Different Core-Shell Structures on the Electrochemical Performances of SiGe Nanorod Arrays as Anodes for Micro-Lithium Ion Batteries. ACS Applied Materials \& Interfaces 2014, 6, 5884-5890.

[18] Hass, R.;García-Cañadas, J.; Garcia-Belmonte, G. Electrochemical impedance analysis of the redox switching hysteresis of poly (3, 4-ethylenedioxythiophene) films. Journal of Electroanalytical Chemistry 2005, 577, 99-105.

[19] Bisquert, J. Chemical capacitance of nanostructured semiconductors: its origin and significance for nanocomposite solar cells. Physical Chemistry Chemical Physics 2003, 5, 5360-5364.

[20] Song, J.; Bazant, M. Z. Effects of nanoparticle geometry and size distribution on diffusion impedance of battery electrodes. Journal of The Electrochemical Society 2013, 160, A15-A24.

[21]Levi, M.; Aurbach, D. Impedance of a single intercalation particle and of nonhomogeneous, multilayered porous composite electrodes for $\mathrm{Li}$-ion batteries. The Journal of Physical Chemistry B 2004, 108, 11693-11703.

[22] Meyers, J. P.;Doyle, M.;Darling, R. M.; Newman, J. The impedance response of a porous electrode composed of intercalation particles. Journal of The Electrochemical Society 2000, 147, 2930-2940.

[23]Xu, C.;Zeng, Y.;Rui, X.;Zhu, J.;Tan, H.;Guerrero, A.;Toribio, J.;Bisquert, J.;Garcia-Belmonte, G.; Yan, Q. Amorphous Iron Oxyhydroxide Nanosheets: Synthesis, Li Storage, and Conversion Reaction Kinetics. The Journal of Physical Chemistry C 
2013, 117, 17462-17469.

[24] Martinez-Julian, F.;Guerrero, A.;Haro, M.;Bisquert, J.;Bresser, D.;Paillard, E.;Passerini, S.; Garcia-Belmonte, G. Probing Lithiation Kinetics of Carbon Coated ZnFe2O4 Nanoparticle Battery Anodes. The Journal of Physical Chemistry C 2014, 118, 6069-6076. 